\title{
THE FINAL RESULTS OF SQUINT OPERATIONS, IN WHICH RESTORATION OF BINOCULAR SINGLE VISION WAS NOT EXPECTED*
}

\author{
BY \\ A. StanWorth \\ MANCHESTER
}

THE purpose of this paper is to analyse the results of operations for cases of squint, in which restoration of binocular single vision was not expected, particularly with regard to the final state of binocular vision.

The clinical material consisted originally of 155 cases treated at the Manchester Royal Eye Hospital, in all of which it was considered that orthoptic treatment was unlikely to benefit the patients, or in which it had already been tried and abandoned. In the latter case the number of treatment sessions had rarely been more than ten, the usual practice in the Orthoptic Department being to give only short courses of treatment if obvious improvement failed to occur (Duthie, 1945). In the interval between the onset of the squint and the institution of orthoptic treatment, correction of the refractive error and any necessary occlusion treatment for amblyopia had been carried out. Of the 155 cases, 21 failed to report for follow-up examination; 26 were excluded because the pre-operative notes were either lost or were equivocal; 4 were excluded because they had received post-operative orthoptic training; and 4 were excluded because the period of time since operation ( 3 months) was too short to assess the final state of binocular vision. There remained 100 cases with satisfactory preand post-operative tests, and the analysis that follows concerns these cases.

\section{Detailed ANALÝsis}

As a result of the synoptophore findings the cases were divided into four groups :-

1. Those in which binocular vision was absent. In these cases, with simple simultaneous perception (S.P.) slides, the two images could only be appreciated simultaneously when they were widely separated, and could not be seen to cross when they were taken past the macular regions, i.e., there was dense central suppression.

2. Those in which simultaneous perception only was present, i.e., in which the two images could be superimposed at the subjective angle.

\footnotetext{
* Received for publication, August 14, 1948.
} 
3. Those in which fusion with amplitude was present.

4. Those in which stereoscopic vision was present.

In order to assess the results more accurately, it has been considered that no appreciable improvement had occurred if the final post-operative reading was still "no binocular vision" or "simultaneous perceeption only" (i.e., Groups 1 and 2), but that if the patient had developed fusion with ductions or stereoscopic vision, then obvious improvement had occurred. The few cases which showed some fusion before operation were excluded, and the 100 cases are divided into 60 with " no binocular vision" pre-operatively, and 40 with " simultaneous perception only."

Of the total of 100,74 showed no appreciable improvement, 26 did improve, 20 developing stereoscopic vision.

Of the 60 with no binocular vision, 51 ( 85 per cent.) did not improve, 9 (15 per cent.) did improve, 7 (12 per cent.) developing stereoscopic vision.

Of the 40 with simultaneous perception, 23 (58 per cent.) did not improve, 17 (42 per cent.) did improve, 13 (32 per cent.) developing stereoscopic vision.

The difference in the percentage improving in the two groups is statistically significant, and the difference is made even more obvious when details of the nine cases improving from " no binocular vision" to' fusion or stereoscopic vision are examined. Of these nine, four had only one pre-operative test, two had shown simultaneous perception several times, but not just prior to the operation, one had false associated fixation, one was a backward child and therefore unreliable, and one developed only weak fusion after operation. Therefore it is probably rare for a case to show the development of fusion or stereoscopic vision purely as a result of operation if binocular vision was originally absent. The prognosis is much better if simultaneous perception was present originally.

\section{The EFfect of Pre-operative Training ON THE RESULTS}

Only 51 of the 100 patients had received pre-operative orthoptic training, though all had been tested on the synoptophore on one or more occasious. No comparison between the trained and the untrained group is worth while, since the former is obviously a highly selected group; but of the 51 patients who had received orthoptic training, all of whom had failed to respond to the training, 16 (32 per cent.) developed fusion or stereopsis after the operation. Twentynine of them had simultaneous perception before operation, and of these, 12 (41 per cent.) improved. Of the 22 with no binocular vision before operation, only 4 (18 per cent.) improved, and even this figure may be too high, as explained above. Nevertheless it 
seems that after operation fusion or stereoscopic vision is developed in one third of the cases in which orthoptic training has produced no improvement.

\section{Relationship of The Results to The Time Since OpERATION}

It is obviously of interest to determine, if possible, the period that elapses between the operation and the recovery of the binocular vision. None of the four patients re-examined within three months of operation had developed fusion or stereoscopic vision, and on the first analysis the results showed that those cases examined within twelve months of operation showed a significantly less percentage of improvement than those examined after more than twelve months. It could be shown, however, that this was due to the former group containing a larger number of cases with no binocular vision originally, and that if Groups 1 and 2 are considered separately, no such significant difference could be found. This suggests that in all probability a patient who is going to develop fusion or.stereoscopic vision will do so within the first twelve months after operation, but a. larger series, especially with repeated post-operative readings, would be needed to substantiate this.

\section{The Effect of The Pre-operative Visúal Acuity .}

All except one of the twenty patients who developed stereoscopic vision had not more than two Snellen lines difference in the visual acuity of the two eyes; the one exception had an acuity of $6 / 36$ in the worse eye, but at one time during treatment for the amblyopia the vision in this eye had been 6/12 partly. Of the six patients who developed fusion, only two had more than two Snellen lines difference in the vision of the two eyes and one of these developed only weak fusion.

In general, then, one can expect improvement only in cases with approximately equal vision; in this type of case, 44 per cent. of those starting with simultaneous perception grades 1 or 2 , and 17 per cent. of those starting with "no binocular vision" improved in their binocular vision. It must again be emphasised that the second figure is probably too high.

\section{The Effect of The Pre-operative Angle}

The average angle of the squint in the cases with no binocular vision pre-operatively was 27 degrees in those which did not improve, and 29 in those which did. 1n those cases originally with . simultaneous perception, the corresponding figure was 23 degrees in each group. It appears then that the size of the pre-operative 
angle of deviation is of no significance as far as the post-operative state of binocular vision is concerned.

\section{Relationship of The Results to The Type OF SQUINT}

Since the patients whose tests are analysed were not seen personally by the writer before operation, and since their notes contained no definite statement by the surgeon in charge as to the type of squint present, it was felt that it would not be worth while to attempt to classify them at this stage. Nevertheless some indication of the types involved can be obtained by correlating the results with the age of the patient at the onset of the squint, the mode of onset, and the refractive error present. 'It is, of course, true that the parent's statements regarding the first two of these factors are not always reliable, but nevertheless the analysis is of some interest.

A definite statement of the age of onset was made in 72 cases; 19 of these developed fusion or stereoscopic vision, and in only two of these was the age of onset less than three years, one being at $2 \frac{1}{2}$ years of age and the other at six months. The latter was particularly interesting. He had never been treated by occlusion, but had developed equal visual acuity in each eye. There was practically no error of refraction present, and he was regarded as a "true alternator." He was operated upon at the age of 7 years and subsequently developed (from no binocular vision) stereoscopic vision. Nevertheless, an early onset of the squint appears to preclude improvement in the binocular vision except in rare cases.

Analysis of the effect of the mode of onset showed that it was in those cases ascribed by the parents to fevers, convulsions, or some form of shock that the greatest number with improved binocular vision occurred, 15 out of 36 (42 per cent.) gaining fusion or stereopsis. Those ascribed to a fall were an exception to this, only one out of ten improving, presumably because this group contains younger children. None of the fourteen cases ascribed to teething or heredity showed any improvement, and they too would obviously fall into a younger age group.

The effect of the refractive error was less definite. No patient with anisometropia of more than 1.50 D.S. developed fusion or stereopsis; of the others, a slightly greater percentage of those with a refractive error of more than $3 \mathrm{D}$, compared with those of under $3 \mathrm{D}$, improved in their binocular state, but the difference ( 37 per cent. compared with 28 per cent.) was not statistically significant.

Correlating all these results, it appears that under these conditions the state of binocular vision in a case of squint of the essential alternating type is unlikely to improve; whereas in cases of the Inilateral type there is a much better chance. 


\section{Relationship to Age at Time of Operation}

In general, the preferred policy has been for operation to be performed at about the age of seven years, after orthoptic treatment has been given a trial. Only one patient in this series was operated on before the age of six years, and that was at the age of $3 \frac{1}{2}$ years. This child developed (from simultaneous perception) full stereoscopic vision. When the remaining cases were divided into five year groups according to their age at operation, no correlation could be found between this and the improvement in binocular vision, those operated on later than the optimum age showing equally good results. The greatest age at the time of operation after which any patient developed stereoscopic vision was nineteen years.

\section{Relationship to The Post-operative Angle}

Of the 40 patients who started with simultaneous perception, 31 had a post-operative angle of deviation of $0-5$ degrees, and of these 16 (49 per cent.) developed fusion or stereoscopic vision. Of the other 9 with more than 5 degrees final angle, only one (11 per cent.) developed fusion, none gaining stereoscopic vision.

Of the 60 patients who started with no, binocular vision 36 had a final angle of $0-5$ degrees, and of these 8 (22 per cent.) developed fusion or stereoscopic vision. Of the remaining 24 , only one (4 per cent.) did so, developing stereoscopic vision.

From this it appears that there is much more chance of developing good binocular vision if the eyes are put really straight. The two patients who obtained good binocular vision with angles of deviation of more than five degrees were both eight degrees divergent, but apparently this was not too great for them to overcome. It may be, however, that the small final angle may be the result rather than the cause of the development of good binocular vision; a decision between these two alternatives might be reached by a comparison of the immediate post-operative readings, taken two weeks or so after the operation, with the final readings. These figures are available in only eight of the cases in which improvement in the binocular state occurred; in these, the average angle immediately after the operation was 7 degrees, the average angle finally being three degrees, suggesting that the smallness of the final angle is the result rather than the cause of the development of good binocular vision.

\section{DişCussion}

These results show that straightening of the eyes results, in an appreciable number of cases, in the development of good binocular vision, even if pre-operative orthoptic training has been ineffective, 
the type of case holding out most prospect of success being one of unilateral squint. with an onset at not less than three years of age, in which the visual acuity has been made approximately equal in the two eyes, and in which simultaneous perception is present. Of this group, which includes 21 patients in the present series, 16 developed fusion or stereopsis after operation, i.e., 76 per cent. Eleven of these patients had received unsuccessful pre-operative orthoptic training. Of the five who failed to improve, two had hyperphoria after the operation, one of 4 degrees and one of 14 degrees, which probably interfered with the development of binocular vision.

The question immediately arises as to whether or not the orthoptic training was sufficiently thorough. It is true that in many orthoptic departments much longer periods of training are given, but it is felt that the results rarely justify submitting the child to the disadvantages of such treatment (Duthie, 1945). In addition, all the cases in this series had shown no sign of progress under treatment, and it is unlikely that any but a very small number would have improved with a longer course.

There are few reported series of cases which can profitably be compared with the present one, since either the cases had not been selected in the same manner, or the operation had been followed by post-operative orthoptic treatment. That a large proportion of adult squints, which are usually considered to be unlikely to develop good binocular vision, can do so following operation has been shown by Shure (1944), 53 per cent. of whose cases aged 18 to 39 years obtained fusion or stereopsis. These patients, however, had postoperative orthoptic training, in view of which it would be interesting to try the effect of such training on a series such as the present one, and see if the final results were improved.

If, in this series in which are selected those cases unlikely to develop good binocular vision, 26 per cent. nevertheless do so after operation, it would be expected that a random series of squints treated, apart from the correction of the refractive error and amblyopia, by operation alone would show an ever higher percentage of good binocular vision. In the series reported by Berens, Elliot and Sobacke (1941), there were 144 cases of this type; 105 of these had no binocular vision or simultaneous perception before operation, and only ten of these ( 9 per cent.) improved their binocular state after operation. This figure is almost the same as the percentage improving due to orthoptic treatment without operation, given in their other series suggesting that had this type of case been removed from their first series, as is done in the present one, there would have been practically no patients who improved in their binocular state after operation. The difference between this and the present 26 percent. improvement is striking and requires explanation. 
The only other obvious difference between the two series is that only the present one had orthoptic treatment before operation, and it may be that this training, although not producing any obvious improvement in their binocular vision, did in some way alter the central nervous pathways so that subsequent improvement in binocular vision was made possible. This may be so, but a comparison of the results in the two other groups reported in the same paper, the difference between which is that only one received pre-operative orthoptic training,-both receiving post-operative training, shows no significant difference between the results when analysed in the same manner as the present series, so that any favourable effect from preoperative treatment such as is postulated above can probably just as easily be obtained from post-operative training.

In addition, such an effect from pre-operative orthoptic training presumably acts by the repeated stimuli through the central nervous system keeping the pathways open by some form of conditioned reflex mechanism, and such an effect might also be achieved by an earlier operation to put the eyes straight. Earlier operation might therefore be considered, not only for this type of cose, but also for those which do show obvious improvement with orthoptic treatment. In this way one could reduce or eliminate the waiting period which often occurs between the correction of the refractive error and and the amblyopia, and the institution of orthoptic treatment, a period which necessitates constant observation and treatment to prevent amblyopia, in which the loss of the stimulation of the central pathways makes subsequent re-education more difficult, and in which there is the possibility of the development of false associated fixation. Early operation is also of decided psychological benefit.

Pre-operative orthoptic training has, of course, its own advantages; a strongly developed fusion faculty before operation will help the eyes to become straight even if the operation corrects the squint only approximately; also one can assess the individual case much more accurately, and nursing at or about the age of seven years is much easier than in a younger child. Nevertheless the end results of such a series of cases operated on as soon as the squint occurs, provided glasses have failed to correct the squint and any amblyopia has been treated, would be of great interest. So far as I am aware, no such series with adequate follow-up examinations has been published, although earlier operation has, of course, been suggested and tried by several authors. Hepburn (1932), for example, thought that better results are obtained if the eyes are put straight before orthoptic treatment is instituted, saying that very little fusion training was then necessary, and stereoscopic vision may be obtained as early as a week after operation. Peter. $(1932,1936)$, preferred operation as soon as possible after the age of three, both in unilateral 
and true alternating squints, and Chavasse (1939) was also a strong advocate of early operation. I Iu the case of the true alternating type of squint, which develops before the age of two years (Wheeler, 1942), and in which in all probablity fusion and stereoscopic vision have never been present, it is likely that operation would have to be within the first two years of life-in order to give much hope of the development of good binocular vision in all except exceptional cases such as the one recorded in the present series.

\section{- Summary}

The state of binocular vision of 100 cases of squint in which restoration of binocular single vision was not anticipated, was assessed post-operatively.

Twenty-six per cent. developed fusion or stereopsis, the favourable factors being :-

(1) The presence of simultaneous perception before operation.

(2) Approximately equal vision in the two eyes.

(3) Age of onset of three years or more, the case being ascribed by the parents to some fever, convulsion, or shock.

(4) A small post-operative angle.

Of the cases fulfilling these criteria 76 per cent. developed fusion or stereopsis, although in most instances unsuccessful pre-operative orthoptic treatment had been given.

It appeared that those patients who were going to develop good binocular vision, did so within one year of operation.

The magnitude of the pre-operative angle of deviation and the age at operation (from six years upwards) was of no significance.

The bearing of these results upon the treatment of squint is discussed, a trial of earlier operation with careful follow-up examinations being suggested.

I wish to express my thanks to the Honorary Surgeons of the Manchester Royal Eye Hospital for permission to study their cases, and especially to $\mathrm{Mr}$. O. M. Duthie for his advice and encouragement; also to Miss E. Stringer and the staff of the Orthoptic Department for the orthoptic examinations.

\section{REFERENCES} Berens, C., Elliot. A. T. and Sobacke, L. (1941).-Amer. J. Ophthal., 24,

Chavasse, F. B. (1939).-Worth's Squint, 7th Ed., 519, 524.

DUTHIE, O. M. (1945).-Brit. Orthoptic J., 3, 13.

HEPBURN, M. (1932).-Trans. Ophthal. Soc. U.K., 52, 366.

Peter, L. C. (1932).-Trans. Ophthal. Soc. U.K., 52, 525.

! (1936). - The Extra-ocular Muscles, 2nd Ed., 205.

ShURE, I. I. (1944).-Amer. Arch. Ophthal., 32, 113.

WHEELER. J. R. (1942).-Trans. Ophthal. Soc. U.K., 62, 351. 